ACTA UNIVERSITATIS LODZIENSIS

Folia Litteraria Romanica 16, 2021

https://doi.org/10.18778/1505-9065.16.24

Marina Tikhonova

Université d'État de Smolensk, Russie

(iD) https://orcid.org/0000-0002-5383-1786

brick_67@bk.ru

\title{
L'amour dans la poésie française contemporaine pour les enfants : entre effusion lyrique et espièglerie argotique
}

\begin{abstract}
RÉSUMÉ
Le thème de l'amour occupe une place importante dans la poésie française pour les enfants, en prenant des aspects très divers. L'amour qui lie parents et enfants est le mieux représenté. Les poètes transmettent cet amour à l'aide de moyens d'expression variés : un lexique affectif, des noms hypocoristiques, le langage enfantin; les tropes (la métaphore, la personnification, etc.) permettent d'exprimer les différentes facettes de l'amour. Les poètes s'inspirent des berceuses françaises traditionnelles. Les poèmes apprennent aux petits lecteurs à choyer leur famille, leurs amis, leur pays natal, la nature, les animaux, la vie. Ce lyrisme n'exclut pas que la poésie pour les jeunes soit empreinte d'humour et d'espièglerie. Pour évoquer les actions des enfants indisciplinés, les poètes recourent à un lexique argotique et familier. Le mélange des registres ajoute au charme de ces poèmes. Les différents visages de l'amour font l'objet d'une riche gamme de moyens d'expression qui reflètent toute la diversité des émotions et des sentiments.
\end{abstract}

MOTS-CLÉS - poésie française contemporaine pour enfants, amour, moyens d'expression.

\section{Love in Contemporary French Children's Poetry: Between Lyrical Effusion and Argotic Playfulness}

\begin{abstract}
SUMMARY
The theme of love occupies an important place in contemporary French children's poetry, being present in very different aspects. The best represented one is love between parents and children. Affective vocabulary, diminutives and childish language indicate a strong emotional interaction between mother and child; tropes (metaphors, personifications etc.) express different facets of love. Poets are sometimes inspired by traditional French lullabies. Full of love, poems teach little readers to appreciate their family, their friends, their homeland, nature, animals, and life. Lyricism, however, does not exclude humour and playfulness. Children love to play and to fool around, and to evoke their mischievous behaviour, poets often recur to slang and familiar vocabulary. The mixture of registers adds to the charm of the poems, whereas a wide range of expressive means reflects the diversity of emotions and feelings.
\end{abstract}

KEYWORDS - contemporary French children's poetry, love, means of expression. 
L'analyse de la poésie française contemporaine pour enfants montre que le thème de l'amour y occupe une place importante, en prenant des aspects très divers. Nous allons les étudier du point de vue de différentes variations de la langue en prêtant attention, entre autres, au lexique des textes poétiques - mots et expressions dont la connotation est vivement exprimée, y compris le lexique affectif et celui qui appartient à l'argot, ainsi qu'au registre qui lui est très proche : le lexique familier et parlé.

Eda Beregovskaya, parlant d'un trait curieux caractérisant le processus de pénétration des mots argotiques dans la langue des belles-lettres, remarque : " À des doses homéopathiques, dans des emplois sporadiques, les argotismes les plus fréquents apparaissent même dans les textes adressés aux enfants [...] » (Beregovskaya, 2011 : 496).

Donc, c'est plutôt un type de lexique rare dans les poèmes pour les jeunes et surtout dans les poèmes liés au sujet que nous abordons dans cet article : «Amour et aimer ». Mais ce lexique y trouve quand même sa place.

La petite quantité de mots ou d'expressions d'argot, d'une part, et le caractère très expressif et ludique du lexique argotique dans le contexte de la poésie pour les enfants, d'autre part, font que le « poids » de l'argot est inversement proportionnel à sa fréquence. Ces mots sont toujours très expressifs et attrayants pour le jeune lecteur, car ils créent souvent un effet de surprise ou de complicité et remplissent une fonction ludique ou plutôt « affecto-ludique » (ou « ludoaffective »), selon les propres termes de Georgette Bensimon-Choukroun (1991: 87).

Passons donc maintenant au thème de l'amour dans la poésie pour les enfants. Avant tout, bien évidemment, c'est l'amour qui lie parents et enfants qui est le mieux représenté. Les poètes transmettent cet amour à l'aide de moyens d'expression variés : le lexique affectif, les suffixes diminutifs et le langage enfantin indiquent une forte interaction émotionnelle, des relations tendres entre la mère (ou le père) et l'enfant. On le voit bien dans le poème de Marcel Béalu « Pour Agnès » que le poète a dédié à sa fille :

Ma fillounnette / Mon bijou rond // Ma vie nouvelle / Mon âme belle // Agnès agneau / Mouton doré // Mon enfant blond / Ma minouchette // Printemps sucré / De mon été // Bourgeon d'azur / Arbre céleste // Ton regard bleu / Comme le ciel bleu // Lave l'instant / De son passé (Malineau, 2004 : 78).

L'amour paternel est exprimé ici à l'aide de toute une série de métaphores évoquant différents éléments de la nature : les animaux (agneau, mouton), les plantes (bourgeon, arbre), les saisons (printemps, été), le ciel (azur, ciel). Ces images sont renforcées par les épithètes rond, belle, nouvelle, doré, sucré, céleste, bleu. Tous ces tropes présentent l'enfant comme un don du ciel, comme quelque chose de très précieux (mon bijou) et unique au monde. 
L'amour du poète et sa tendresse se font également sentir grâce à deux mots à forte valeur affective : fillounnette (synonyme affectueux du mot fille ou fillette en langage parental) et minouchette (synonyme affectueux du chat ou minou en langage enfantin).

Les poètes contemporains s'inspirent aussi parfois des berceuses françaises traditionnelles qui nous emmènent au cœur de la petite enfance et de l'amour maternel intarissable. Dans le poème « Pour dormir » de Jacques Charpentreau, des tropes tels que la métaphore et la personnification permettent à l'auteur de créer une image poétique très expressive de cet amour :

[...] Ferme tes yeux, mon ange, dors, / Voici le pays des merveilles, / Mon papillon, ma tendre abeille, / De fleur en fleur prends ton essor, / Ton âme vole, et moi je veille. // Je ne sais plus quel est ton sort. / Es-tu l'oiseau qui vole libre ? / Es-tu le chaton ou le tigre ? / Dans les songes, loin de ton corps, / Tu t'échappes. Ton âme émigre. [...] (Charpentreau, $2004: 53$ ).

La mère veille sur son enfant en l'appelant affectueusement mon trésor, mon ange. Elle protège son sommeil fragile qui l'emporte dans un pays mystérieux et énigmatique où l'enfant devient un papillon, une abeille, un oiseau (qui vole libre), un chaton ou un tigre. Il faut dire qu'un des traits caractéristiques de la poésie pour enfants est lié au monde des animaux.

La poésie pour les enfants accompagne avec tendresse et douceur tous les moments de la vie des petites filles et des petits garçons. Ces poèmes pleins d'amour apprennent aux petits lecteurs à choyer leur famille, leurs amis, leur pays natal, la nature, les saisons, les animaux, la vie...

Citons à titre d'exemple trois textes du poète belge francophone du $\mathrm{XX}^{\mathrm{e}}$ siècle Maurice Carême. En caractérisant son œuvre, Jacques Charles a remarqué que les thèmes de Maurice Carême se réduisent au fond à un seul : l'amour (Charles, 1965 : 36). Mais l'amour dans la poésie de Maurice Carême n'est pas quelque chose d'abstrait.

Le premier de ces thèmes, c'est l'amour de la nature. Le poète écoute « de très secrets murmures de la nature, il déchiffre mille signes visibles et invisibles qui n'ont que pour lui seul voix délibérative » (Charles, $1965: 36$ ). Pour le poète, « retrouver les bois, les moissons, les banquises de nuages, le cliquetis des eaux vives, c'est revenir au " paradis » de ses premiers éveils, c'est boire à la source originelle» (Charles, $1965: 40)$.

Par exemple, le poème de Maurice Carême « Pommelette » est empli de la tendresse et en même temps de la pitié que le poète ressent pour une petite pomme qui repose dans un panier et qui sera bientôt mangée :

Dis, qu'as-tu, pommelette, / À faire la dormette / Au fond de ton panier ? / Aurais-tu oublié / Le bon vent du verger / Qui te faisait danser ? // Préfères-tu, seulette, / Qu'on te porte au marché / Où tu seras croquée ? / Dis, qu'as-tu, pommelette, / À faire la dormette / Au fond de ton panier? (Carême, $1976: 59$ ). 
Le suffixe -ette sert ici à construire les noms diminutifs et hypocoristiques pommelette, dormette, seulette qui expriment une intention caressante, affectueuse, ce qui est fréquent, notamment dans le langage des enfants ou ses imitations.

L'héroïne du poème " J'ai six ans » parle de sa vie de tous les jours : de l'école, de ses occupations préférées, de sa tortue, de son frère aîné, des moments gais ou tristes de sa vie. Ce récit nous replonge dans notre propre enfance et nous rend un peu nostalgiques. Et nous comprenons, avec la petite héroïne de Maurice Carême, que le bonheur est fait de ces petites choses quotidiennes, de ce que nous aimons et de ceux qui nous aiment, et que l'amour donne un sens à notre vie :

J'ai six ans. Je vais à l'école. / Déjà, je puis écrire " vole » / Avec une plume d'oiseau. / J'aime les gâteaux, mais tout chauds. / Les jours de pluie, je m'habitue / À dire TU à ma tortue, / Car je ne l'appelle jamais / Que madame de Tortulet. // Je joue plus souvent qu'à mon tour / Avec la flûte et le tambour / De mon frère aîné Évariste. / Il arrive que je sois triste / Après avoir été plus gaie / Que la grive dans le pommier. / Est-ce pour cela que j'existe ? / Je dis cela sans y penser, / C'est peut-être ça, EXISTER (Carême, 1976 : 151).

Le thème de la mère occupe une place très importante dans l'œuvre de Maurice Carême. Le poète a beaucoup aimé sa mère et il lui a consacré des pages très émouvantes. Le poème « Pour ma mère » illustre l'amour filial de l'enfant qui ne sait pas exprimer bien ses sentiments et qui ne se rend pas encore compte de ce qu'il ressent. Remarquons que le poète n'emploie pas ici les mots amour ou aimer, mais il recourt à l'hyperbole pour montrer l'immensité et la force de ce sentiment :

Il y a plus de fleurs / Pour ma mère, en mon cœur, / Que dans tous les vergers ; / Plus de merles rieurs / Pour ma mère, en mon cœur, / Que dans le monde entier ; / Et bien plus de baisers / Pour ma mère, en mon cœur, / Qu'on en pourrait donner (Carême, 1976 : 42).

Ce lyrisme n'exclut pas que la poésie pour les jeunes soit empreinte d'humour et d'espièglerie. Les enfants aiment jouer et faire des bêtises et, pour évoquer les actions des enfants indisciplinés ou des garçons qui tombent amoureux des filles, les poètes recourent parfois au lexique argotique et familier en adéquation avec le caractère très expressif et ludique de l'enfant désobéissant. Le mélange des registres ajoute au charme de ces poèmes.

Ainsi, Jacques Charpentreau introduit une expression familière dans son poème « Incompris » dont la narration se fait à la première personne. Le narrateur, un écolier, veut plaire à une fille de sa classe. Pour cela, il met des cafards dans son capuchon, vide son tube de colle dans son cou... Pourtant toutes les tentatives du garçon amoureux se soldent par un échec et il avoue : J'ai pris ma claque (prendre une claque 'subir un échec' (Colin et al., 2010 : 194)) :

Ce matin, allant à l'école, / J'ai mis quatre cafards d'un coup / Dans le capuchon de Nicole : / Elle n'a pas aimé du tout. // J'ai pressé mon tube de colle / Et je l'ai vidé dans son cou / 
Avec trois ou quatre bricoles, / Des gommes, des crayons, des clous. // Je n'ai pas su plaire à Nicole. / Nous n'avons pas les mêmes goûts / M'a-t-elle dit sans protocole : / J'ai pris ma claque, un point, c'est tout (Charpentreau, $1986: 35$ ).

Parfois le lexique argotique se regroupe dans des séries synonymiques. Dans le poème « Des goûts et des couleurs », Jacques Charpentreau nous présente différents personnages dont chacun a ses goûts et ses intérêts. La notion d'amour renvoie ici à une grande variété de sentiments, d'états et de comportements, allant d'un plaisir général lié à un objet concret (la crème au chocolat, les chapeaux cloches, l'horloge parlante, etc.) ou à une activité (photographier les étoiles filantes, chercher les cris des vieux films d'épouvante et d'autres), ou encore une attirance profonde pour une personne (par exemple, Roméo aimant Juliette). Pour exprimer cette diversité de sentiments, le poète introduit une chaîne synonymique de sept verbes et expressions successifs qui ont le sens de aimer et constituent une gradation : être affolé, aimer, avoir le béguin (qui vient justement de l'argot où le béguin signifie 'amour sincère, mais peu motivé' ou 'amour non vénal' (Colin et al., 2010 : 59)), adorer, chérir, idôlatrer, être pris par :

Bertrand est affolé de crème au chocolat, / Mon amie Isabelle aime les chapeaux-cloches / Et Jean-Louis a toujours des bonbons dans ses poches. / Ce sont les salsifis que cherche Nicolas. / Bernard photographie les étoiles filantes, / Jean collectionne les billets de tombola. / Moi, j'aime l'horloge parlante. // Françoise a le béguin pour les voix de ténor, / Chez Hector-Saturnin, les Walkyries chevauchent, / Ses haut-parleurs les font passer de droite à gauche, / Suzette aime l'éclat de sa Rolls-Royce en or, / Julien cherche les cris des vieux films d'épouvante, / Alfred le son du cor, Félix le vent du nord. / Moi, j'aime l'horloge parlante. [...] (Charpentreau, $1984:$ 32-33).

Donc, comme nous avons pu voir, les personnages de la poésie pour les enfants peuvent ressentir de l'amour pour des choses bien variées.

Très souvent, les poètes attribuent des propriétés humaines à un animal ou à une chose inanimée. Dans les poèmes pour les enfants qui parlent de l'amour, la personnification aide à créer des situations comiques et même paradoxales. D'habitude ces poèmes sont pleins d'humour ou d'ironie. Par exemple, Jean-Luc Moreau parle de l'amour entre un soulier et une chaussure. Le soulier propose à la chaussure de se marier, mais la chaussure est très capricieuse et le soulier finit par se révolter (le poète recourt ici au verbe argotique se rebiffer) et il épouse la botte :

Le soulier dit à la chaussure : / «Allons, ne te fais pas prier. / Même couleur, même pointure : / nous devrions nous marier. » / La belle fait la difficile ; / elle en rêve mais s'en défend ; / si bien que lui, se rebiffant : / «Tu me prends pour un imbécile ? » // Il épousa la botte et lui fit douze enfants (Moreau, 2003 : 91).

Les personnages du poème " Géométrie » du même auteur sont encore plus originaux. Il s'agit de deux droites parallèles qui s'aiment depuis longtemps mais 
ne peuvent même pas se toucher car, selon les lois géométriques, les deux parallèles ne se rencontrent pas, ou, comme on dit, elles se joignent à l'infini. En écoutant leurs plaintes, le poète s'indigne de leur indécision, leur conseille d'essayer de se rapprocher quand même et lance un juron (que diable !) qui manifeste justement son indignation et qui pousse les deux parallèles à franchir le pas décisif :

Deux droites parallèles / depuis longtemps s'aimaient : / Nous toucher, disaient-elles, / le pourrons-nous jamais ? / Messieurs les géomètres / nous parlent d'infini ; / c'est bien beau de promettre, / mais tant de kilomètres, / ça donne le tournis !... // - Si le sort vous accable, / leur répondis-je alors, / rapprochez-vous, que diable, / rapprochez-vous encor ! // Ma remarque, opportune, / leur fut d'un grand secours : / il n'en reste plus qu'une. / Quel beau roman d'amour! (Moreau, $1992: 124)$.

Mais la poésie enfantine connaît également des histoires d'amour tragiques. Tel est l'amour entre l'allumette et le cigare dans le poème de Jean-Luc Moreau, où le poète, sous forme humoristique, montre « le dangereux visage de l'amour » : l'amour qui peut détruire :

La petite allumette, / aimant un gros cigare, / rêva d'un rendez-vous, / vit son rêve aboutir // et sut en s'éteignant / que l'amour nous égare... / Un seul baiser de feu / peut nous anéantir (Moreau, 1992 : 133).

Parmi les poèmes étudiés, on trouve des textes qui produisent un effet de surprise ménagé par l'auteur à la fin du texte, qui éclaire son sens et peut conduire à le réinterpréter. La tonalité recherchée est le plus souvent humoristique.

Cet ingénieux artifice de composition permet à Jacques Charpentreau d'embrouiller les lecteurs. Le titre du poème, «À celle que j' aime», et le texte même font penser à une vraie déclaration d'amour dans laquelle le héros chante la beauté de sa bien-aimée : sa grâce, sa voix, son teint. Tout en elle suscite son regard admiratif, le ravissement. Le poète introduit un lexique appartenant au champ sémantique de l'amour : le verbe aimer à la première personne du singulier qui se répète six fois, les substantifs envie et passion. Différentes épithètes attribuées à l'objet de l'amour (vif, argentin, jolie, coquette, charmante) ajoutent de nouveaux traits expressifs au portrait de la bien-aimée en complétant son image idéale. Dans cette description, on remarque également une métaphorisation quand le héros parle des rayons et des feux de son amour, et se présente comme le prisonnier de sa chaîne.

Et seule la fin du poème dévoile le vrai objet de l'amour du héros lyrique :

J'aime tes courbes et tes lignes, / La grâce de ton col de cygne, / J'aime tes rayons et tes feux / Quand nous partons seuls tous les deux / Et que nous suscitons l'envie ; / J'aime le cadre de ta vie, / J'aime l'éclat vif de ton teint / Et j'aime ton timbre argentin ; / Je suis prisonnier de ta chaîne, / Je te guide, mais tu me mènes, / Je t'aime toute avec passion / Et jusqu'aux dents de tes pignons, / Toi, si jolie et si coquette, / Ô ma charmante bicyclette ! (Charpentreau, $1984: 116$ ). 
Cette conclusion inattendue est préparée pour permettre au lecteur vigilant de deviner peu à peu la signification du texte. On fera, par exemple, attention à des mots tels que le col, le cadre, les rayons, les feux, la chaîne, les dents, le pignon qui désignent les composants de la bicyclette, des pièces qui sont facilement identifiables. L'emploi de ces mots dans leur sens métaphorique et concret en même temps, c'est-à-dire une syllepse, aide l'auteur à créer cette ambiguïté, à jouer sur le double sens. D'autre part, l'apparition du mot-clé est dictée ici par la loi de la rime qui établit un lien entre les mots coquette et bicyclette.

Donc, notre analyse a montré que, dans la poésie française contemporaine pour les enfants, les différents visages de l'amour font l'objet d'une riche gamme de moyens d'expression qui reflètent, notamment grâce au recours à un lexique à valeur affective, expressive y compris familier et argotique, toute la diversité des émotions et des sentiments de l'enfant et de l'adulte. Le ton des poèmes qui parlent de l'amour va du lyrisme à l'espièglerie et à l'humour, ce qui correspond très bien au caractère ludique de la poésie pour les enfants.

\section{Bibliographie}

BENSIMON-CHOUKROUN, Georgette (1991), «Les mots de connivence des jeunes en institution scolaire : entre argot ubuesque et argot commun $»$, Langue française, $n^{\circ}$ 90, p. 80-94

BEREGOVSKAYA, Eda (2011), «L'argot français : évolution de sa perception » in Registres de langue et argot(s). Lieux d'émergence, vecteurs de diffusion (S. Bastian, J.-P. Goudaillier éds), München, Éditions Martin Meidenbauer, p. 487-502

CHARLES, Jacques (1965), Maurice Carême, Paris, Éditions Seghers

COLIN, Jean-Paul, MÉVEL, Jean-Pierre, LECLÈRE, Christian (2010), Dictionnaire de l'argot et du français populaire, Paris, Larousse

\section{Corpus}

CARÊME, Maurice (1976), Poèmes pour petits enfants, Paris, Librairie Hachette CHARPENTREAU, Jacques (1984), La poésie dans tous ses états, Paris, Les Éditions Ouvrières CHARPENTREAU, Jacques (1986), La Banane à la moutarde, Paris, Nathan CHARPENTREAU, Jacques (2004), La carpe dans mon pommier, Paris, La Maison de Poésie MALINEAU, Jean-Hugues (2004), Premiers poèmes pour toute ma vie, Toulouse, Éditions Milan MOREAU, Jean-Luc (1992), Poèmes de la souris verte, Paris, Hachette MOREAU, Jean-Luc (2003), Poèmes à saute-mouton, Paris, Hachette Livre

Marina Tikhonova - linguiste, docteure ès lettres, maître de conférences au département de français de l'Université d'État de Smolensk (Russie). Ses domaines de recherche sont la stylistique, l'analyse du texte littéraire, la littérature, en particulier la poésie française contemporaine pour les enfants, la traduction et la didactique. 